\title{
On derivation of Dresselhaus spin-splitting Hamiltonians in one-dimensional electron systems
}

\author{
I.A. Kokurin $1,2,3$, Ж \\ ${ }^{1}$ Institute of Physics and Chemistry, Mordovia State University, 430005 Saransk, Russia \\ ${ }^{2}$ Ioffe Institute, 194021 St. Petersburg, Russia \\ ${ }^{3}$ St. Petersburg Electrotechnical University "LETI", 197022 St. Petersburg, Russia
}

(Dated: January 3, 2019)

\begin{abstract}
Two-dimensional (2D) semiconductor structures of materials without inversion center (e.g. zincblende $\mathrm{A}^{\mathrm{III}} \mathrm{B}^{\mathrm{V}}$ ) possess the zero-field conduction band spin-splitting (Dresselhaus term), which is linear and cubic in wavevector $k$, that arises from cubic in $k$ splitting in a bulk material. At low carrier concentration the cubic term is usually negligible. However, if we will be interested in the following dimensional quantization (in $2 \mathrm{D}$ plane) and the character width in this direction is comparable with the width of 2D-structure, then we have to take into account $k^{3}$-terms as well (even at low concentrations), that after quantization leads to comparable contribution that arises from $k$-linear term. We propose the general procedure for derivation of Dresselhaus spin-splitting Hamiltonian applicable for any curvilinear 1D-structures. The simple examples for the cases of a quantum wire (QWr) and a quantum ring (QR) defined in usual [001]-grown 2D-structure are presented.
\end{abstract}

\section{INTRODUCTION}

The spin-orbit splitting of conduction band plays a key role in various effects, such as: (i) Dyakonov-Perel spin relaxation [1, 2]; (ii) photo-galvanic effects [3], (iii) current-induced spin polarization (see for instance recent overview [4] and references therein); (iv) intrinsic spin Hall effect [5] and some others. In low-dimensional structures there are three principal mechanisms of spin-orbit coupling (SOC): (i) structure inversion asymmetry (SIA) or Rashba term [6] that is due to asymmetry of a heterostructure potential profile; (ii) bulk inversion asymmetry (BIA) or Dresselhaus term [2] arising due to the lack of inversion center in a bulk material [7]; and (iii) interface inversion asymmetry (IIA) leading to a renormalization of the BIA contribution [8]. Here we will discuss BIA term in 1D system defined in 2D-structure by electrostatic gating or etching, e.g. quantum wire (QWr), quantum ring $(\mathrm{QR})$ or more complex curvilinear $1 \mathrm{D}$ structures.

The conduction band spin-splitting in bulk $\mathrm{A}^{\mathrm{III}} \mathrm{B}^{\mathrm{V}}$ material is cubic in momentum, and it is given by [7]

$$
H_{\mathrm{BIA}}=\gamma \boldsymbol{\sigma} \kappa,
$$

where $\boldsymbol{\sigma}=\left(\sigma_{x}, \sigma_{y}, \sigma_{z}\right)$ is the vector of the Pauli matrices, $\kappa_{x}=k_{x}\left(k_{y}^{2}-k_{z}^{2}\right)$, and $\kappa_{y}, \kappa_{z}$ can be found by cyclic permutations. It should be noted, that such a form of Dresselhaus Hamiltonian is true for the reference frame with $x\|[100], y\|[010], z \|[001]$.

The Dresselhaus SOC for quantum well (QW) can be found from Eq. (10) by averaging on a wave-function $\psi_{0}(z)$ of a ground state of the quantized motion

$$
H_{\mathrm{BIA}}^{2 \mathrm{D}}=\beta_{z}\left(\sigma_{y} k_{y}-\sigma_{x} k_{x}\right)+\gamma\left(\sigma_{x} k_{x} k_{y}^{2}-\sigma_{y} k_{y} k_{x}^{2}\right),
$$

\footnotetext{
*E-mail kokurinia@math.mrsu.ru
}

where $\beta_{z}=\gamma\left\langle k_{z}^{2}\right\rangle$, with $\left\langle k_{z}^{2}\right\rangle=\left\langle\psi_{0}\left|k_{z}^{2}\right| \psi_{0}\right\rangle$. Frequently, at low concentration due to inequality $k_{x, y} \ll\left\langle k_{z}^{2}\right\rangle \simeq$ $\left(\pi / w_{z}\right)^{2}$ only the first $k$-liner term in Eq. (2) is taken into account, with $w_{z}$ being the width of $2 \mathrm{D}$-layer. These results are derived for [001]-grown QW.

If a cubic in $k$ term in Eq. (2) is neglected, then it leads to some misunderstanding when one tries to derive Dresselhaus Hamiltonian for the structure of the lower dimension based on $2 \mathrm{D}$ one, e.g. a quantum wire. The strict procedure means the use of the bulk Dresselhaus term (11) as a starting point with the quantization (and corresponding averaging) in two directions. For QWr defined in [001]-grown 2D-system and with transport direction along [100] this leads to

$$
H_{\mathrm{BIA}}^{1 \mathrm{D}}=\left(\beta_{y}-\beta_{z}\right) \sigma_{x} k_{x}
$$

where $\beta_{y}=\gamma\left\langle k_{y}^{2}\right\rangle \simeq \gamma\left(\pi / w_{y}\right)^{2}$ with $w_{y}$ being the $\mathrm{QWr}$ width in the plane of initial 2D-structure. The use of $k$-linear term only as staring point leads in this case to the same result, but with $\beta_{y}=0$. This is true for QWrs which width $w_{y}$ is much wider than $w_{z}$, but the condition of the single subband filling (one-dimensionality) holds. Thus, the result that is obtained from the $k$-linear operator differs only by strength parameter. But it is worth noting, that in QWr of the square cross-section there is no spin-splitting due to $\beta_{z}=\beta_{y}$. It means that the $k$ linear Dresselhaus term is not enough, especially good it can be seen for structures with other crystallographic orientation, both the orientation of initial 2D-structure and the QWr orientation in 2D plane.

\section{GENERAL APPROACH FOR DERIVATION OF BIA HAMILTONIAN IN ONE DIMENSION}

Using the simple example of QWr we shown that in general the $k$-linear SOC Hamiltonian is poor starting 

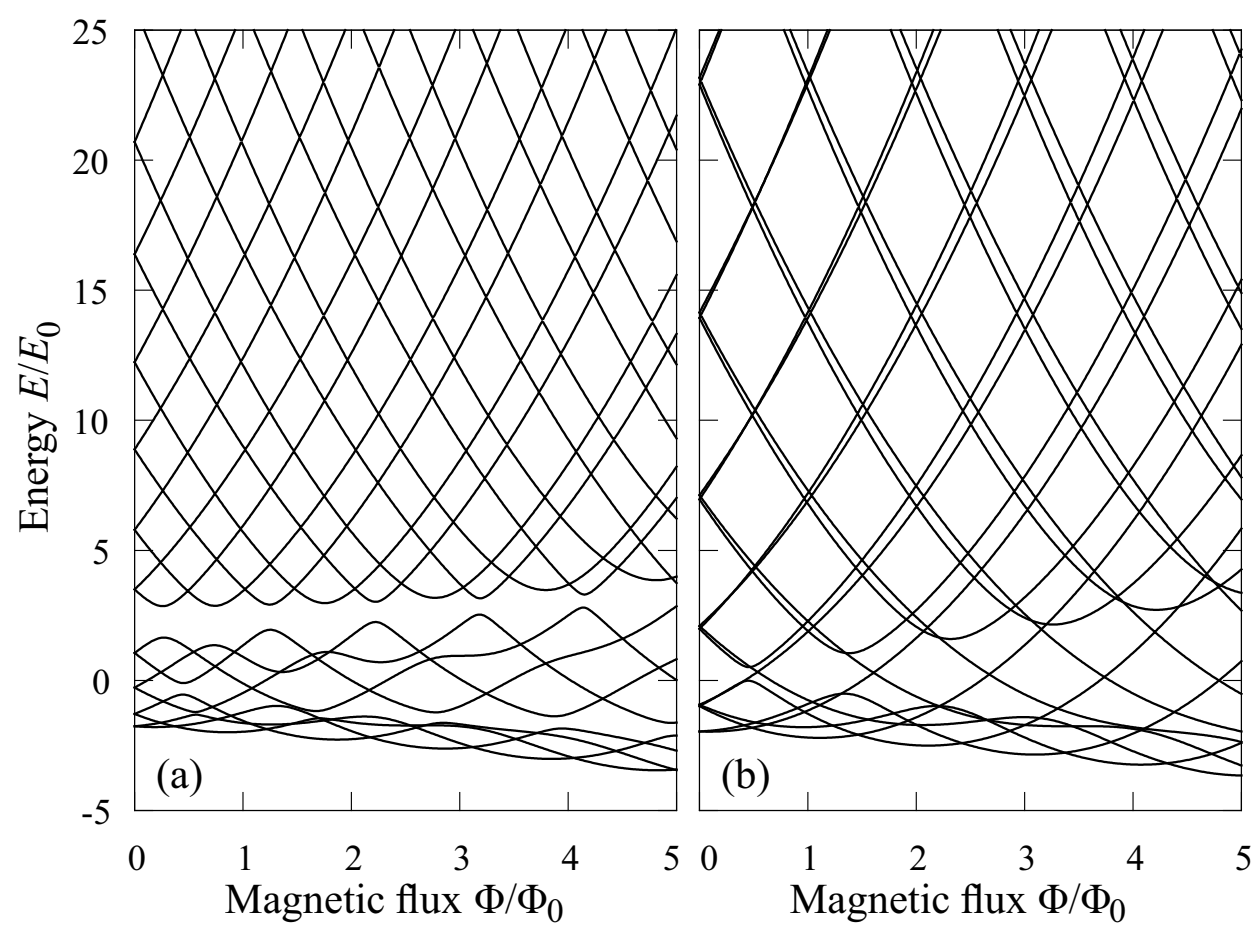

FIG. 1: The energy levels of QR with Dresselhaus SOC as a function of magnetic flux. Parameters are following: $g=12.0$, $m^{*} / m_{0}=0.05,2 \beta_{z} m^{*} r_{0} / \hbar^{2}=2.8, E_{0}=\hbar^{2} / 2 m^{*} r_{0}^{2}$. (a) Total BIA contribution are taken into account, $\beta_{r}=\beta_{z} / 2,(\mathrm{~b})$ analytical result for the simplified model with $\beta_{r}=0$.

point at dimension lowering. Method of invariants [9] permits to find the form of BIA Hamiltonians from symmetry consideration for certain structure with defined crystallographic orientation. However, the strength of entering parameters and the relationship between them can be found only by means of strict procedure starting from bulk BIA-splitting. But for curvilinear structures, such as QR, the strict procedure based on a ground-state averaging, is much more complicated.

We propose the method of the effective spin-orbit Hamiltonian derivation based on our previous work [10], where we used a simple approach. Our approach includes the following steps: (i) the derivation of bulk BIA Hamiltonian for necessary crystallographic orientation using the coordinate transformation; (ii) the dimension lowering (to 2D) of a bulk Hamiltonian (an averaging on a ground-state wave function of the transverse motion, $z$-direction) with conservation of high-power terms in $k$; (iii) an additional rotation of coordinates (around $z$-axis on $\varphi$ angle) that with simultaneous quantization (averaging) in new $x$-direction leads to BIA Hamiltonian for 1D QWr that orientation coincides with the tangential direction to point with curvilinear coordinates $(\xi, \zeta)$; (iv) the replacement $\sigma_{x} \rightarrow \sigma_{\xi}, \sigma_{y} \rightarrow \sigma_{\zeta}, k_{y} \rightarrow k_{\zeta}$ with the following symmetrization of non-commuting terms (i.e. $A B \rightarrow\{A, B\}=(A B+B A) / 2)$, since Pauli matrices $\sigma_{\xi}$ and $\sigma_{\zeta}$ in a 'new' reference frame in general do not commute with remaining momentum operator $k_{\zeta}$.

\section{BIA HAMILTONIAN FOR THIN QUANTUM RING}

Let us apply above scheme to the derivation of BIA Hamiltonian for thin (1D) circular QR of radius $r_{0}$ defined in a usual 2D-structure with [001]-orientation. Two first points in our scheme can be omitted, and we start from Hamiltonian (2). After rotating of coordinates and $x$-quantization we have to replace $\sigma_{x}$ and $\sigma_{y}$ by $\sigma_{r}(\varphi)=\cos \varphi \sigma_{x}+\sin \varphi \sigma_{y}, \sigma_{\varphi}(\varphi)=-\sin \varphi \sigma_{x}+\cos \varphi \sigma_{y}$, respectively. Remaining momentum $k_{y}$ has to be replaced by $K_{\varphi}=r_{0}^{-1}\left(-i \partial / \partial \varphi+\Phi / \Phi_{0}\right)$, where we include in consideration the magnetic field $\mathbf{B}$ with the flux $\Phi=\pi r_{0}^{2} B$ and flux quantum $\Phi_{0}=2 \pi \hbar c /|e|$. After symmetrization we find

$$
\begin{gathered}
H_{\mathrm{BIA}}^{\mathrm{ring}}=\left(\beta_{z}-\beta_{r}\right) \cos 2 \varphi \sigma_{\varphi} K_{\varphi}+\left(\beta_{z}+\frac{\beta_{r}}{2}\right) \sin 2 \varphi \sigma_{r} K_{\varphi} \\
+\left(\beta_{z}-\frac{5}{2} \beta_{r}\right) \frac{i}{2 r_{0}} \sin 2 \varphi \sigma_{\varphi}-\left(\beta_{z}+2 \beta_{r}\right) \frac{i}{2 r_{0}} \cos 2 \varphi \sigma_{r},(4)
\end{gathered}
$$

where $\beta_{r} \simeq \gamma\left(\pi / w_{r}\right)^{2}$, with $w_{r}$ being the radial width of the ring.

If the $\mathrm{QR}$ radial width is much wider than its axial width $\left(w_{r} \gg w_{z}\right)$ then the inequality $\beta_{z} \gg \beta_{r}$ is fulfilled and neglecting terms containing $\beta_{r}$ in Eq. (4) we find after some algebra the following operator

$$
H=\beta_{z}\left[\sigma_{\varphi}(-\varphi) K_{\varphi}-\frac{i}{2 r_{0}} \sigma_{r}(-\varphi)\right]
$$


that was derived in Ref. [11] taking into account only $k$-linear terms in BIA SOC of initial 2D Hamiltonian.

Now consider the total Hamiltonian containing a kinetic term $\hbar^{2} K_{\varphi}^{2} / 2 m^{*}\left(m^{*}\right.$ is the electron effective mass), the Zeeman splitting $H_{Z}=(1 / 2) g \mu_{B} B \sigma_{z}$ ( $g$ is the effective g-factor and $\mu_{B}$ is the Bohr magneton) and BIA SOC from Eq. (44). Such a Hamiltonian can be diagonalized only numerically. One can utilize any appropriate basis set, e.g. $\Psi_{m s}(\varphi)=(2 \pi)^{-1 / 2} e^{i m \varphi} \chi_{s}$, with $m=0, \pm 1, \pm 2, \ldots$ and $\chi_{+1}=\left(\begin{array}{l}1 \\ 0\end{array}\right), \chi_{-1}=\left(\begin{array}{l}0 \\ 1\end{array}\right)$. The results of a numerical diagonalization are depicted in Fig. 1a. The reduced Hamiltonian matrix $102 \times 102$ $(m=0, \pm 1, \ldots, \pm 25$ and $s= \pm 1)$ gives perfect precision for plotted levels. One can see a significant difference in Fig. 1b, where the simplified BIA term was used $\left(\beta_{r}=\right.$ 0) [11]. In this case the spectral problem can be solved analytically due to the fact that Hamiltonian commutes with the following operator, $f_{z}=-i \hbar \partial / \partial \varphi-(\hbar / 2) \sigma_{z}$.

\section{CONCLUSIONS}

In conclusion, we have shown that the use of $k$-linear 2D BIA-term as a starting point is not enough for derivation of correct 1D BIA Hamiltonian. We proposed the approach of the SOC Hamiltonian derivation for any $1 \mathrm{D}$ structure that defined in a 2D-system from a material without the inversion center. The BIA Hamiltonian for thin QR oriented in (001)-plane is constructed. This Hamiltonian can be used to calculate the persistent charge and spin currents using the equilibrium density matrix formalism [12].
[1] M.I. Dyakonov, V.I. Perel, Spin relaxation of conduction electrons in noncentrosymmetric semiconductors. Sov. Phys. Solid State 13, 3023 (1972).

[2] M.I. Dyakonov, V.Yu. Kachorovskii, Spin relaxation of two-dimensional electrons in non-centrosymmetric semiconductors. Sov. Phys. Semicond. 20, 110 (1986).

[3] E.L. Ivchenko, S.D. Ganichev, Spin-Photogalvanics, in Spin Physics in Semiconductors, ed. by M.I. Dyakonov (Berlin, Springer, 2008) pp. 245-277. doi:10.1007/978-3-540-78820-1_9.

[4] N.S. Averkiev, I.A. Kokurin, Current-induced spin orientation in semiconductors and low-dimensional structures. J. Magn. Magn. Mater. 440, 157 (2017). doi:10.1016/j.jmmm.2016.12.059.

[5] J. Sinova, D. Culcer, Q. Niu, N.A. Sinitsyn, T. Jungwirth, A.H. MacDonald, Universal Intrinsic Spin Hall Effect. Phys. Rev. Lett. 92, 126603 (2004). doi:10.1103/PhysRevLett.92.126603

[6] Yu.A. Bychkov, E.I. Rashba, Properties of a 2D electron gas with lifted spectral degeneracy. JETP Lett. 39, 78 (1984).
[7] G. Dresselhaus, Spin-Orbit Coupling Effects in Zinc Blende Structures. Phys. Rev. 100, 580 (1955). doi:10.1103/PhysRev.100.580

[8] U. Rössler, J. Keinz, Microscopic interface asymmetry and spin-splitting of electron subbands in semiconductor quantum structures. Solid State Commun. 121, 313 (2002). doi:10.1016/S0038-1098(02)00023-6

[9] E.L. Ivchenko, G.E. Pikus. Superlattices and Other Heterostructures. Symmetry and Optical Phenomena (Berlin, Springer, 1997). doi:10.1007/978-3-642-60650-2

[10] I.A. Kokurin, Effect of spin-orbit coupling on spectral and transport properties of tubular electron gas in InAs nanowires. Physica E (Amsterdam) 74, 264 (2015). doi:10.1016/j.physe.2015.07.003

[11] J.S. Sheng, K. Chang, Spin states and persistent currents in mesoscopic rings: Spin-orbit interactions. Phys. Rev. B 74, 235315 (2006). doi:10.1103/PhysRevB.74.235315.

[12] I.A. Kokurin, Electronic States and Persistent Currents in Nanowire Quantum Ring. Semiconductors 52, 535 (2018). doi:10.1134/S1063782618040188 